\title{
Exploring Effectiveness of Formative Assessment for Music Students in China
}

\author{
Zijun Deng \\ ${ }^{1}$ University College London, Grower Street, London WC1E 6BT, UK \\ ${ }^{a}$ Emaile:dtnvzde@ucl.ac.uk
}

\begin{abstract}
In China, teachers are continually involved in the implementation of learning assessment policy, an implication of how they perceive the necessity of the purposes and functions of assessment. This qualitative study paper explores the effectiveness of formative assessment of music students in China. The paper begins by providing an overview of formative assessment of music, describing the nature and purpose of formative assessment. It also examines what do Chinese people value in music and what can be assessed, where affective, psychomotor, and cognitive areas of musical intelligence are identified. These are regarded as ideas that assessment develops the positive personal qualities and learning capabilities of learners to a more negatively perceived duty of assessment for learning management and inspection. This allows for better insights into the glitches policymakers may experience while trying to thwart the negative consequences linked with high-stakes examination systems in China.
\end{abstract}

Keywords: Formative assessment, summative assessment, music assessment.

\section{INTRODUCTION}

The organic process of reorganizing and restructuring as students learn provides an alternative to the old model of teaching and learning process. According to Gipps [11]. This approach provides learning in terms of networks with links in many directions rather than an external map that is directly transposed into the heads of learners. The contemporary approach to teaching and learning embodies a change to "how to learn" from "what to learn". According to the humanistic-constructivist concept of education, the old model of teaching knowledge into learners' minds offers an avenue to the method of knowledge creation in the mind. In this aspect, the focus of teaching and learning is not to quantitatively pile facts but to actively and qualitatively comprehend the concepts and the links that exist among them. The practice of educating students to actively, creatively, and independently learn encompasses their social, esthetical, emotional, intellectual, and physical reactions. In this aspect, learning to understand implies the development of intelligence that is appropriate to solve problems encountered in real-life settings. As defined by Gardner [10], intelligence is the aptitude to provide solutions to glitches or to model products that have value within cultural settings. Based on his concept of multiple intelligences, there is a sequence of intelligence that each can be explored in a vast range of education extents such as linguistic, musical, interpersonal, and intrapersonal intelligence, just to name a few.

There is a link between music education and other intelligence. According to Gardner [10], it is increasingly difficult to deny the notion of the existence of at least some intelligence and the comparative independence of each other which can be formed and integrated into a diversity of adaptive ways by both individuals and cultures. As such for balanced learning development, it is necessary to consider all the intelligence. Thus, this represents a suitable basis on which formative assessment within music learning can be anchored. According to the concept of humanistic-constructivism, formative assessment forms a vital portion of the process of learning. This is particularly because by its very definition, formative assessment is inclined towards the process of learning as it encompasses understanding about learners prevailing talents and thoughts and acknowledging the development level attained and the appropriate steps to take next [15]. It fosters the recognition of the quality of the prevailing state of education and captures recommendations for continued learning growth, therefore it presents vital feedback to both the learner and the teacher as pertains to present understanding and skill growth that are vital in determining the way into the future. 
The Chinese society educational system is an examination driven providing discontent with the educational outcomes of the system [7]. However, the country has been committed to reducing the focus on external summative examinations as the key assessment objective. This paper explores the practice of formative assessment as the most appropriate assessment approach for Music Students in China.

\section{UNDERSTANDING ASSESSMENT OF MUSIC}

FORMATIVE

According to Black and William [5], there is an interconnection between music teaching, learning and assessment processes. A teacher can more effectively design and check the suitability of the procedures used in music teaching by observing music learning processes while paying special attention to the learners' nature of work such as interest, learning tactics, individuality, orientations, help, and stimulation among others. In this aspect, Harlen and James [15], argue that pupil referenced formative assessment provides a way to judge the work of learners or progress by taking into consideration such aspects as the determination they put in, the specific context of the learners' work, and the process attained by the learners over time (p.370). The basis of formative assessment of music learning is the methodical and qualitative reflection based on the standards arising from musical goals of creating, playing, and singing. As an aspect referred to as the criterionreferenced facet of formative assessment.

Formative assessment of music learning implies making decisions as pertains to the importance of the learners' musical development based on the planned music goals while taking into account the original level of the learners' music growth. It offers an opinion on the music learning process quality. The opinion that learners get in the learning process assists them to recognize what they are doing wrong or right, why is it right or wrong, what learning approaches can assist them to enhance their music successes as well as learn about their strengths and weaknesses [23]. However, above all, it provides motivation, self-validation, and self-assurance in their aptitudes. Music assessment is a vital part of the teaching-learning process. This is because it is inclined to both the learning results and the procedure. As a process, assessment recognizes the learner's distinctive way of sensation, rational and performing music. In this aspect, assessment can be perceived as not only validation or rejection of music accomplishments, but as procedure excellence with diagnostic, formative, informative, and evaluative roles.

Music learning formative assessment is conducted based on specific standards that stem from the purpose of teaching music and which mirror the qualitative degree of accomplishing the set musical goals. In this aspect, the standards need to vividly and realistically outline the most vital and valuable aspects of musical accomplishments [23]. Systematically, they should provide answers to questions about areas in which learners should make progress and answer questions about areas in which children should advance and musical capabilities and skills they need to develop about their psychomotor, emotional, and intellectual growth. Additionally, the standards should point out what learners are needed to develop and in terms of what topographies will form the basis for judging their music accomplishments. Moreover, the learners must be conversant with the standards, understand them, and their capacity to provide justice to their work. Learners should become experienced assessors of their learning growth. According to Gipps [11], in addition to understanding what counts as the criteria expected of their assessment, learners need sustained experience in ways of inquisitive and enhancing the value of their work and supported experience in assessing their work. Based on such musical standards, learners can discover answers to selfevaluation questions such as what is it that I do not like? What have I learned? What problems do I have? What do I have to change? How do I feel? [6] [1]

\section{WHAT DO PEOPLE VALUE IN MUSIC AND WHAT CAN BE ASSESSED?}

Formative assessment of music learning can be assessed in affective, psychomotor, and cognitive areas of musical intelligence [23]. Formative assessment of music learning in the affective sphere assesses musical intelligence about intrapersonal and interpersonal intelligence of learners. According to Gardner [10], looking at music as the intelligence that is contained in sound is particularly a process of interpersonal and intrapersonal with both interpersonal and intrapersonal intelligence. Music can reach areas of the personality structure that are often overlooked in the modern school such as growth and awareness of both emotional and social life as a requirement for complete life as well as deterrence against lack of emotional adaptability, despair, lacking communication, hostility, and disquiet. This is particularly because music is known to provide a successful remedy in such instances. In this aspect, music should be linked with other aesthetic topics as an important area in the school program. According to Gardner [10], the growth of logical-mathematical and linguistic intelligence in the modern-day intensely prevails over other intelligence like musical, spatial, interpersonal, bodily-kinesthetic, and intrapersonal. The result is that such unbalanced growth of intelligence, therefore, threatens a balanced structure of personality growth which in turn further threatens the future of human growth in modern civilization.

In learning, affective factors are very vital not only for music. Even though there is the assumption of an individual eagerness to learn in most present 
psychological analyses, such factors like appropriate motivation, a set of values that favors a specific type of learning an affective state accompanying learning and a reassuring cultural setting are essential educational and assessment factors [15]. Musical knowledge path is cemented with both personal and interpersonal intelligence such that by creating, performing, and listening, learners develop such important things as positive self-image, positive feeling in connection to music, attentiveness, aesthetic thoughtfulness, and selfconfidence just to name but a few. Under this criteria, learners' musical intelligence such as interest, expression, emotions, spontaneity, and attentiveness are important musical values that can be assessed [23].

Formative assessment in music learning in the area of psychomotor assesses musical intelligence about bodilykinesthetic and multi-dimensional intelligence and musical intelligence about etymological and logicalmathematical intelligence. Given that learners cannot develop musical aptitudes, talents, and knowledge without actively performing, it implies the critical interconnectedness between music learning and psychomotor experience. The majority of musical activities specifically singing expression of musical experience with movement and art and instrument playing are part of psychomotor goals. Motor response to music is perceived to be dominant especially during the earliest period of learners' development. For example, in the early primary-school period, it still prevails about art or other verbal communication. As such, effective music teaching and assessment approaches use an action as a component to form and prompt concepts of sound. Gardner [10] argues that music is best perceived as a comprehensive gesture in form of movement or direction that is conducted implicitly with the body. In this aspect, given that learners naturally relate to music and body movement, it forms enough basis to integrate movement into processes of music teaching, learning, and assessment. Planning of psychomotor music goals is vital especially in influencing the formation of sound concepts of learners. This is because, in the presence of movement, learners can concretize the theoretical nature of musical constraints [18]. In which case they develop the appropriate knowledge and understanding for the transfer of musical involvement and conceptions into motor communication. The interaction between music intelligence and spatial bodily-kinesthetic intelligence fosters the development of body awareness, vocal techniques, motor abilities, and motor sensitivity and evaluation [23]. Under this principle, learners' musical intelligence such as performing skills, coordination, types of moves, move expression, verbal articulation is important for assessment.

On the other hand, formative assessment of music learning in the cognitive area takes into account musical intelligence about linguistic and logical-mathematical intelligence. As musical experiences grow gradually, they form musical concepts that characterize the foundation for musical rationality. In this aspect, musical knowledge takes into account all processes of recording audio, memorizing, and implement the concepts in fresh settings of music creation and evaluation. Therefore, while the affective area of musical experience forms the foundation, the cognitive area of musical experience is the stimulation for performing. This makes the connection between affective and cognitive areas not only mutual but also interdependent. According to Bamberger [4], laying the basis on the audial perception to understanding what material is heard paths to cognitive processes that can provide better sense to the renowned musical phenomena. To think and understand musically means to provide meaning to a musical perception by igniting appropriate sound concepts that are modeled in the process of listening, creating, and performing. Thus, direct musical experience results in musical knowledge which forms a foundation on which learners can create musical capabilities and skills. Learners can develop intuition, imagination, and memorization, thought strategies, communication in diverse areas of expression, critical evaluation, and attentiveness through musical intelligence interaction with logical-mathematical and linguistic intelligence. Under this standard, learners' musical intelligence such as expressive qualities, performance accuracy, tonal stability, musical memory, recognition of sound characteristics, and performance accuracy can be assessed [23].

While learners' intelligence about music such as the general knowledge about music, knowledge of the how of music such as maintaining a regular pulse, and knowledge of music that is attained through immersion in the musical experience and activities that pathway to a deeper understanding of the unique expressive character of the music is the key are valued music intelligence, the last type of intelligence forms the main reason why people engage with music. Though the other kinds of music intelligence can anchor and enrich learners' music knowledge, they are not self-sufficient and as such need not be taught and assessed in isolation [21].

\section{UNDERSTANDING WHO ASSESS MUSIC, WHO SHOULD ASSESS MUSIC?}

The Music Curriculum Guide (CDC) [17] provides that learners are expected to develop skills, knowledge, and positive attitudes as key learning objectives by integrating music activities of creation, listening, and performing. This is achieved through means of appropriate learning, teaching, and assessment [17]. The guide also provides a list of modes of assessment that music teachers can utilize to assess their students. According to the guide, music teachers are expected to assess learners' performance in music creation, performing for both singing and playing instruments and listening [17], using varied assessment approaches. 
These include the use of worksheets, practical tests, concert reports, project learning, classroom performance, self and peer assessment, portfolio, and music activities record. In line with other study subjects in the classroom, music teachers must report learners' performance twice every year.

\section{OVERVIEW OF CHINESE MUSIC TEACHERS PRACTICE}

The 2001 New Curriculum Reform in China had a notable effect on the education of China [13]. Before the introduction of the new curriculum, music teachers in the country transmitted knowledge to learners through direct instruction with a primary focus on academic results. However, in the new curriculum, music teachers are required to incorporate music with other study subjects. Additionally, the new curriculum allows teachers to focus on students' music learning processes rather than on the results, therefore fosters the capabilities of a student to develop key skills like critical and creative thinking [13]. The main objective of the new music education curriculum was to strike a balance between the interests of learners as well as cultivate their critical thinking and problem-solving capacities and inspire learners in their musical creativity. Distinct from the prior curriculum, the new curriculum encourages music teachers to integrate music creation activities with traditional singing and listening [26].

Also, the new curriculum and teaching materials encourage a more open and diversified music learning setting that has a key goal of modeling a music education that is more resourceful and enjoyable for learners. This is because the new era is entrenched in the aesthetic music qualities and structured based on students' level of grades. For instance, according to Guo [13], the curriculum recommends music teachers to use music games and musical knowledge to teach learners in grades one and two. On the other hand, students in grades three up to six are taught instrumental performance and forms of music. Teachers for these grades are also expected to assist learners to experience the emotions that underlie the music they study and listen to. For learners from grades seven to nine, music serves to educate and model their passion for music where music teachers reduce singing activities as a result of changes in boys' voices to focus on music appreciation through such aspects as listening and analysis of music [19]. At the advanced level of the new curriculum, music teachers use advanced technology to teach music in an environment that is more open and diversified that focuses on cultivating learners' music creativity. Music teachers at this level, therefore, are required to possess higher levels of musical capabilities and creative approaches to teachings.

Unlike in the previous music curriculums, where music teachers' obligations were to bring musical understanding and educate learners on how to sing in the confines of a single and formalized learning structure, the new curriculum has new educational concepts and challenges for music teachers. According to Feng [9], music teachers now face an increased workload as they are now required to meet the new teaching requirements. Additionally, the new curriculum has brought a shift from the old teacher-centered education system that was more examination-oriented to a system that also emphasizes the need for practice and creation where learners are allowed to explore and experience a variety of musical elements on their own rather than solely relying on their educators [13]. According to Ho [16], four major objectives underpin the new music curriculum; integration of learners' experiences with the individualism of the modern popular culture, to continue to educate traditional moral values, cultivation of learners patriotic and nationalistic spirit, and facilitation of multiculturalism that bring an appreciation of the global culture. Zhu [25] argues that besides, the new curriculum has an objective to model cognition, moral and aesthetic values in learners. This implies a shift from the contentbased to experience-based kind of teaching and learning music [20].

For the teachers, the new curriculum is perceived to be more flexible as it provides teachers with guidance on such aspects as music appreciation, perception, performance, creation, and culture-linked music. On the other hand, it encourages learners to learn and play musical instruments that are not only relevant in the modern-day culture but also appeal to them. This requires educators to have an open mind in adopting prevailing music pedagogical philosophies [19].

\section{SELF-REFLECTION AND SELF- ASSESSMENT}

Music educators should strive to assist learners to become independent singers and performers with the capacity to critique their learning, performance, and work and to make enhancements that are based on the feedback provided [24]. Students shared responsibility for listening, critiquing, and reviewing can be modeled through such classroom assessment strategies that task learners in providing feedback to others and themselves. This, therefore, can assist learners to take up substantial independence and control over their learning. Andrade and Cizek [2], put to test several formative classroomcentered assessment approaches such as students as an important feedback source all through the learning procedure. Additionally, several scholars have provided guidelines that draw on learners as foundations of feedback throughout the assessment procedure [12]; [14]; [8]. However, there are a few models that offer unequivocal direction on how music teachers can engage learners' informative music assessment procedures. Moreover, much fewer exemplars of appropriate tools that provoke the kind of information that can result in 
advanced intensities of learning and better instructional decisions exist [24].

\section{CONSIDERATION FOR SUMMATIVE APPROACH}

Conventionally, selection forms one of the basic objectives for summative assessment. Assessment in music education is perceived by many to be the formal examinations such as the A-Levels or GCSEs, examinations for instrumental and vocal grading, or music competitions and auditions. The results of such assessments are then utilized by others directly especially for auditions or indirectly where examination marks or grades were used for assessment, to decide who should progress to bands, choirs, or universities. All these examinations and other assessments like tests issued to learners at the end of the term or examinations at the end of the year marking the end of a particular project or module are key examples of summative assessment. Summative assessment in music provides a snapshot of what learners have attained in their learning at a specific point in time as compared to specific and preset standards [22]. At its best, it is an assessment that reflects on what learners have attained by showing what they know, understand, or can do as pertain music learning.

\section{CONCLUSION}

Musical attainments result from the processes of learning music that happens throughout music activities of performing, listening, and creating. Assessment should therefore follow or better form part of the practical ways in which such aspects are represented. Growing the musical knowledge of young people should be at the center of music education in both the way teachers assess learners and what they assess. According to Gardner [10], rather than bring the learners to assessment like the case of psychometricians, assessment should be taken to the learners by modeling an environment with appealing resources that allow learners to exhibit their spectra of music intelligence in a way that is as natural as possible. Drawing on the modern music learning approaches, music assessment is procedure orientated. As such, formative music assessment that happens throughout performing, listening, and creating music can directly reflect on the affective, psychomotor, and cognitive intelligence of learners' musical development. This is because, formative assessment as a process orientated, deploys flexible approaches of assessing musical attainments rather than using set standards on aptitudes and knowledge examinations. The formative assessment measures are devised to assess what is least essential in the arts [3]. Moreover, rather than testing musical knowledge by observing the evoked cortical responses during listening to pure tones, focuses on educating learners to sing, play instruments or create and change melodies, therefore, providing a good ground to gauge how well learners have mastered these skills [10]. Formative assessment provides both flexibility and complexity in methods of observing, testing and assessing, and providing feedback on the music teaching and learning process quality. In addition to providing a diagnosis of the prevailing state of the learning process, it also provides both the prognosis and propositions for additional effort. Therefore, it has the function of promoting the learning procedure and shaping selfreflection. When conducted in an appropriate and positive atmosphere, it provides a basis to look for music intelligence in learners and not the absence of it.

\section{REFERENCES}

[1] Andersson, T. D., Armbrecht, J., \& Lundberg, E. (2012). Estimating use and non-use values of a music festival. Scandinavian Journal of Hospitality and Tourism, 12(3), 215-231.

[2] Andrade, H., \& Cizek, G. J. (Eds.). (2010). Handbook of formative assessment. Routledge.

[3] Aróstegui, J. L. (2003). On the nature of knowledge: What we want and what we get with measurement in music education. International Journal of Music Education, (1), 100-115.

[4] Bamberger, J. S. (1995). The mind behind the musical ear: How children develop musical intelligence. Harvard University Press.

[5] Black, P., \& Wiliam, D. (1998). Assessment and classroom learning. Assessment in Education: principles, policy \& practice, 5(1), 7-74.

[6] Blacking, J. (1969). The value of music in human experience. Yearbook of the International Folk Music Council, 1, 33-71.

[7] Brown, G. T., \& Gao, L. (2015). Chinese teachers' conceptions of assessment for and of learning: Six competing and complementary purposes. Cogent Education, 2(1), 993836.

[8] Burrack, F. (2002). Enhanced assessment in instrumental programs. Music Educators Journal, 88(6), 27-32.

[9] Feng, D. (2006). China's Recent Curriculum Reform: Progress and Problems. Planning and Changing, 37, 131-144.

[10] Gardner, H. E. (2000). Intelligence reframed: Multiple intelligences for the 21 st century. Hachette UK.

[11] Gipps, C. V. (1994). Beyond testing: Towards a theory of educational assessment. Psychology Press. 
[12] Goolsby, T. W. (1999). Assesment in Instrumental Music: How can band, orchestra, and instrumental ensemble directors best assess their student's learning? Here are some evaluation tools and techniques to consider. Music educators journal, 86(2), 31-50.

[13] Guo, L. (2012). New curriculum reform in China and its impact on teachers. Comparative and International Education, 41(2).

[14] Hale, C. L., \& Green, S. K. (2009). Six key principles for music assessment. Music Educators Journal, 95(4), 27-31.

[15] Harlen, W., \& James, M. (1997). Assessment and learning: differences and relationships between formative and summative assessment. Assessment in education: Principles, policy \& practice, 4(3), 365-379.

[16] Ho, W. C. (2010). Moral education in China's music education: Development and challenges. International Journal of Music Education, 28(1), 71-87.

[17] Hong Kong Curriculum Development Council (CDC). (2003). Music curriculum guide (Primary 1Secondary 3).

[18] Kelly, G. J., \& Chen, C. (1999). The sound of music: Constructing science as sociocultural practices through oral and written discourse. Journal of Research in Science Teaching: The Official Journal of the National Association for Research in Science Teaching, 36(8), 883-915.

[19] Law, W. W., \& Ho, W. C. (2009). Globalization, values education, and school music education in China. Journal of Curriculum Studies, 41(4), 501520 .

[20] Leung, B. W., \& McPherson, G. E. (2010). Students' motivation in studying music: The Hong Kong context. Research Studies in Music Education, 32(2), 155-168.

[21] Russell, J. A., \& Austin, J. R. (2010). Assessment practices of secondary music teachers. Journal of research in music education, 58(1), 37-54.

[22] Scott, S. J. (2012). Rethinking the roles of assessment in music education. Music Educators Journal, 98(3), 31-35.

[23] Sicherl-Kafol, B. (2005). Formative assessment in music education-an overview of Slovenian primary school theory and practice.

[24] Valle, C., Andrade, H., Palma, M., \& Hefferen, J. (2016). Applications of peer and self-assessment in music education. Music Educators' Journal, 102(4), 41-49.

[25] Xiaoman, Z. (2006). Moral education and values education in curriculum reform in China. Frontiers of education in China, 1(2), 191-200.

[26] Xie, J., \& Leung, B. W. (2011). Students' motivation to study music: The mainland China context. Research Studies in Music Education, 33(1), 59-72. 